\title{
Using Digital Resources for the ECE Curriculum in China: Current Needs and Future Development
}

\author{
Jing Zhou*
}

Faculty of Early Education and Special Education,

East China Normal University, No. 3663 North Zhongshan Road,

Shanghai, China

E-mail: jzhou@spe.ecnu.edu.cn

*Corresponding author

\section{Si Chen}

Faculty of Early Education and Special Education,

East China Normal University, No. 3663 North Zhongshan Road,

Shanghai, China

E-mail: april19850422@hotmail.com

\section{Lixian Jin}

Division of Speech and Language Therapy, School of Allied Health Sciences, De Montfort University, Leicester LE1 9BH, UK

E-mail: jin@dmu.ac.uk

\begin{abstract}
Using digital resources is an important development in the Early Childhood Education (ECE) curriculum in China. Guo and Wang (2005) report that $98 \%$ of urban ECE programs have computers with Internet connections, in addition to other technical facilities, which are used daily in ECE classrooms. However, the lack of curriculum-related digital resources and of a network to share them makes it difficult for teachers to share these resources for teaching. Further, this development of digital resources for ECE should consider Chinese cultures of learning (Jin \& Cortazzi, 2006) in order to meet the needs of Chinese learners and maximize the learning effect. This paper focuses on the major features of digital resources in Chinese ECE and a framework for developing the content through examining existing digital resources and materials. Methods of inquiry and evaluation include the use of focus groups of kindergarten teachers in different provinces in China.

The findings (Chen \& Zhou, 2009; Zhou \& Chen, 2009) indicate that an ECE digital resource should have features of individualization, interaction, sharing and sociability in networking in Chinese educational contexts, for supporting teaching design, practice, evaluation and reflection. An effective framework of ECE digital resources is recommended to contain three key parts:
\end{abstract}

1) A Teacher Planning System to support teachers' information searches, classified according to themes, subjects or types of activities.

2) A Children's Learning System to offer interactive learning at school or home following the classroom curriculum.

3) A Family Support System to involve parents in their children's learning and development. 
Keywords: Digital Resources, Chinese ECE Curriculum, learning effect.

Biographical notes: Jing Zhou is Professor of Early Years Education and Deputy Dean in the Faculty of Early Education and Special Education, East China Normal University. She is the principal investigator of the research project on Digital Resource for Chinese Kindergarten Curriculums from the Chinese Minister of Education. Her publications include more than a dozen books and 80 papers in journals and books, plus many conference proceeding papers. Professor Zhou has served in editorial positions for Chinese early education journals as well as some international journals. Her B.A. (Chinese Language), M.A. (Child Development), and Ph.D. (Education) degrees are from Nanjing Normal University in China, Connecticut College in the U.S.A. and the University of Hong Kong.

$\mathrm{Si}$ Chen is a postgraduate student in the Faculty of Early Education and Special Education, East China Normal University. She majors in Early Years Education and was a key contributor for this research project on digital resource for Chinese kindergarten curriculums. Her research interests include early literacy curriculum in Chinese kindergarten and using digital resources for the early reading curriculum in China.

Lixian Jin is Reader in Linguistics and Health Communication and the Director for the Centre of Intercultural Research in Communication and Learning (CIRCL) at De Montfort University, UK. Her research and publications into Chinese and other learners of English and investigating language development and clinical assessments of Chinese speaking children have been widely recognized by researchers and language teachers both in the West and in Asia. She is regularly invited to give public lectures, key-note speeches and workshops to international and professional audiences. In Britain she has served as a member of the E-learning Partnership Board for the Higher Education Funding Council of England (HEFCE) and a member of the HEFCE Assessment Panel for $e$-UK-China program. She is an executive editorial board member or the review editor for a number of international journals in the UK, U.S.A. and Hong Kong.

\section{Introduction}

The reform of the early childhood education (ECE) curriculum in China in the $21^{\text {st }}$ century demonstrates a trend that teaching focuses now on holistic education rather than on the teaching of separate subjects. A key issue to sustain this development and enhance teaching is the construction of Chinese ECE curriculum resources. The use of digital resources for the ECE curriculum is a key aspect of this resource issue. However there was little research based information available in this aspect for the Chinese ECE until the Study of Digital Resources for Chinese Kindergarten Curriculum, sponsored by the Humanity and Social Sciences Research Fund of the Chinese Ministry of Education (Zhou \& Chen, 2009). This paper discusses functions, applications and needs of the digital resources for the Chinese ECE curriculum and raises issues for future development. 


\section{Current Situations and Challenges}

There are two key issues for the development of digital resources applied to the Chinese ECE curriculum: the use of digital resources for the ECE teaching and the construction of an ECE corpus for teaching and learning.

In recent years, the use of information technology has become a norm in urban kindergartens. This means many city kindergartens now use computers and the Internet for teaching in addition to the use of overhead projectors, digital cameras, recorders, etc. In Shanghai, Guo and Wang (2005) have investigated the use of information technology in 99 kindergartens which include the standard four types of kindergartens in China: Model Kindergartens, Kindergartens of the First Rank, the Second Rank and Private Kindergartens. The findings showed that $98 \%$ of these kindergartens were equipped with computers, although they were used mainly by teachers.

These teachers are able to use the Internet competently, e.g. to search information on such websites as Baidu, Google, Yahoo, Sohu; as well as to use some educational websites for collecting teaching materials, such as the website of The Shanghai ECE (Fu, 2008). They also use the Internet to share and exchange information and teaching plans and discuss them with teachers in other kindergartens to generate further ideas and to enhance their teaching.

However, a common practice of using computers in kindergartens is that teachers predominantly use word-processing facilities (Guo \& Qian, 2006). Further, the educational websites available are not specific enough for Chinese ECE, thus teachers may have to devote a lot of time to search for information from different non-specialized websites, which does not help share information with other teachers. Some relevant websites tend to have the same or similar information, e.g. the same PowerPoint slides or pictures, videos. Given the common time constraints, it is unlikely that these teachers will recreate the content from these websites, which defeats the purpose of using internet resources. In summary, the following challenges exist for the development of Chinese ECE e-resources.

1. There is a lack of specialized ECE websites for Chinese kindergarten teaching and learning; some ECE websites contain few individual and specialized features, since websites tend to share similar information in both content and form.

2. The presentations of some websites do not support teachers to obtain useful information and apply to their teaching, since these websites appear to group different things on the same webpage. The information is too varied and the content is broad. It is too time-consuming for teachers to select relevant materials for their teaching purposes.

3. Some websites do not offer effective technical support, which makes it difficult for users to manage the use of websites.

4. There is a need to develop e-resources which reflect local teaching and learning standards and yet also have features which can easily be shared with other places. In short, it is crucial now for ECE teachers in China to have curriculum related eresources to share for their teaching.

The Chinese Ministry of Education published the Kindergarten Education Guidance (2002), which challenged the teachers to develop a holistic education environment for kindergarten children. This document emphasizes that this phase of education needs to be inspiring, curriculum subjects need to be integrated and classroom teaching should be flexible with multiple-theme activities. These emphases require and encourage ECE 
teachers to change their traditional ways of teaching and to teach more creatively in class: this means that outside the class they need to access new teaching materials and resources for their preparation of the classroom teaching. However, kindergarten teachers have been facing difficulties of shortages of relevant new curriculum resources. It has been argued (Yan \& Fan, 2005) that there should be three types of curriculum e-resources established for the ECE reform and to overcome such challenges: in-kindergarten teaching e-resources, outside-kindergarten (but local) e-learning resources and a large scale and well supported web resource. With such a structure available, the teaching activities in kindergarten can become more varied, more interactive, integrated, inspiring and can fit the new requirements of Chinese ECE teaching and learning. Nevertheless, teachers find themselves greatly constrained to develop this structure on their own, due to such difficulties as the unevenness of resources available in different parts of the country and the problem of ensuring the quality and accuracy of online information. Further, it is not suitable to just copy Western models of curriculum e-resources. Rather, it is essential for Chinese teachers to develop our own e-resources for Chinese children based on Chinese cultures of learning and Chinese educational features (Jin \& Cortazzi, 2006; 2009).

The current study focuses on investigating how Chinese kindergarten e-resources have been established and used, what features are needed in the construction of eresources for the Chinese ECE curriculum and how e-resources based on Chinese cultures of learning support the development of ECE teachers.

\section{Methods and Procedure of the Study}

A multiple set of methods is used for this study, including formulating a needs analysis from ECE teachers, making classroom visits and observations, and conducting interviews and focus group discussions with 47 kindergarten teachers in Shanghai and in Zhejiang and Sichuan provinces. Data were collected from transcribed interviews and discussions as well as through written reports by these teachers. A main reason for selecting kindergarten teachers from these three areas is that these three represent three levels of economic development in China, i.e. advanced development (Shanghai), average development (Zhejiang Province) and a relatively disadvantaged stage of development (Sichuan Province). Also different types of kindergartens were chosen for this study. These were state and private kindergartens; and - hierarchically - kindergartens from different recognized ranks: National Model kindergartens, Provincial Model kindergartens and those of the First Rank and Second Rank. Teachers interviewed and in focus group discussions include those who have had many years of teaching as well as those who had up to five years of teaching. This coverage thus gives a good spread of types of kindergartens, contrasting areas and teachers, which supports the reliability of the data and findings.

The procedure for this study had three steps:

1. Conducting a needs investigation and analysis;

2. Arranging focus group discussions with two group meetings; these were held in Shanghai with invited kindergarten teachers and ECE specialists from East China Normal University to talk about their ideas, comments and suggestions to the construction of an ECE curriculum e-teaching corpus.

3. Conducting individual interviews and classroom observations to obtain feedback from teachers who have tried to use existing e-resources; this was done in order 
to evaluate the strengths and weaknesses of particular e-resources and to find out what elements a corpus of ECE e-resources needs to contain. This sequenced procedure has helped the researchers gather information, evaluations and reflections from different angles and dimensions.

\section{Research Findings and Discussion}

There are two main aspects of the findings: the needs analysis demonstrates the necessity of using ECE e-resources for curriculum teaching and learning in the digital era; and second the identification of key elements for the development of an effective corpus of eresources for the Chinese ECE curriculum.

\subsection{The Establishment of a Corpus of E-Resources Needed for the Chinese ECE Curriculum}

The use of information technology (IT) should be considered as an integrated part of the ECE curriculum development. As a tool, medium and a method, IT used for teaching should be evaluated at all levels including the preparation of teaching activities, the teaching-learning process and learning assessment (Guo \& Wang, 2005; Guo \& Qian, 2006).

The findings from all the interviews show that the IT needs are urgently and widely demanded by ECE teachers for curriculum-related e-resources, because of the recent teaching requirement of using an open, individually-tailored and holistic approach, which makes it quite impossible to use the traditional teaching of using standard provided and prepared teaching materials. This strongly suggests that teachers have to spend a large quantity of time to prepare to meet the individual needs of children and to facilitate the development of children's creativity and imagination in learning. ECE teachers in Sichuan province report that multi-media technology is a useful support for their teaching. It helps young children overcome their comprehension difficulties for some abstract concepts. When e-resources are available online, it is more convenient for teachers to access information and to guide the children in their learning. Some teachers confirmed that young children become more independent in learning with the help of internet resources. It is important that these e-resources should be uniquely oriented towards young children. They found that when the e-resources fit with needs and features of young children, the learners become more interested in learning and understand newly introduced concepts better. Their senses were more holistically stimulated so that their listening, looking and doing were integrated.

A traditional use of e-resources mainly has benefits of easy and fast access, an economical cost and the effectiveness of digitalization and Internet availability. However, this may not encourage users to be creative. With the development of science and technology, the Internet will provide interaction and a non-centralized service. The new corpus of e-resources for Chinese ECE should constantly update the use of Internet technology and enhance the management of knowledge and information services.

An updated Internet service of ECE e-resources should provide a variety of teaching and learning materials, by using IT to collect and classify these resources and materials. To allow for local circumstances and to meet individual children's needs, teachers need to be given a range of choices for teaching in different contexts. The quantity of these specialized e-resources should therefore be of a sufficient size that teachers can select materials to match the individual needs of learners. Teachers can use the Internet to share and store their own e-teaching materials and build up their personal corpus of materials. 
With this much broader knowledge of materials and teaching approaches they can compare and evaluate their own teaching through their own assessments and reflection. Further, the function of websites is to provide a sharing platform for teachers and parents to exchange information and ideas for their children's learning and to monitor their progress more effectively.

\subsection{The Structure and Value of the ECE Corpus of E-Resources}

The teachers were questioned what key elements should be included in the ECE corpus of e-resources. The participants strongly suggest that such a corpus should offer teachers convenience of access and a broader variety of choices of teaching materials and information; and provide children with more learning opportunities, greater flexibility in the use of learning time and a richer study environment. The teachers and focus group participants indicate that this ECE corpus of e-resources has to be practical and realistic for early years' education. The corpus also should consider catering for the parents of young children so that it facilitates the interaction between young children and adults. To produce this corpus, it is essential to establish a team with both technical specialists and ECE experts so that it maximizes the application of the expertise of both groups of professionals. Based on the data from interviews, focus group discussions, classroom observations, teachers' evaluative reports and needs analysis, the key elements include individuality, interactivity, sharability and sociality for the use of e-resources. Thus the corpus should have three types of e-systems: a Teaching Support System, a Children Learning System and a Family Support System for learning and education at home (see Figure 1).

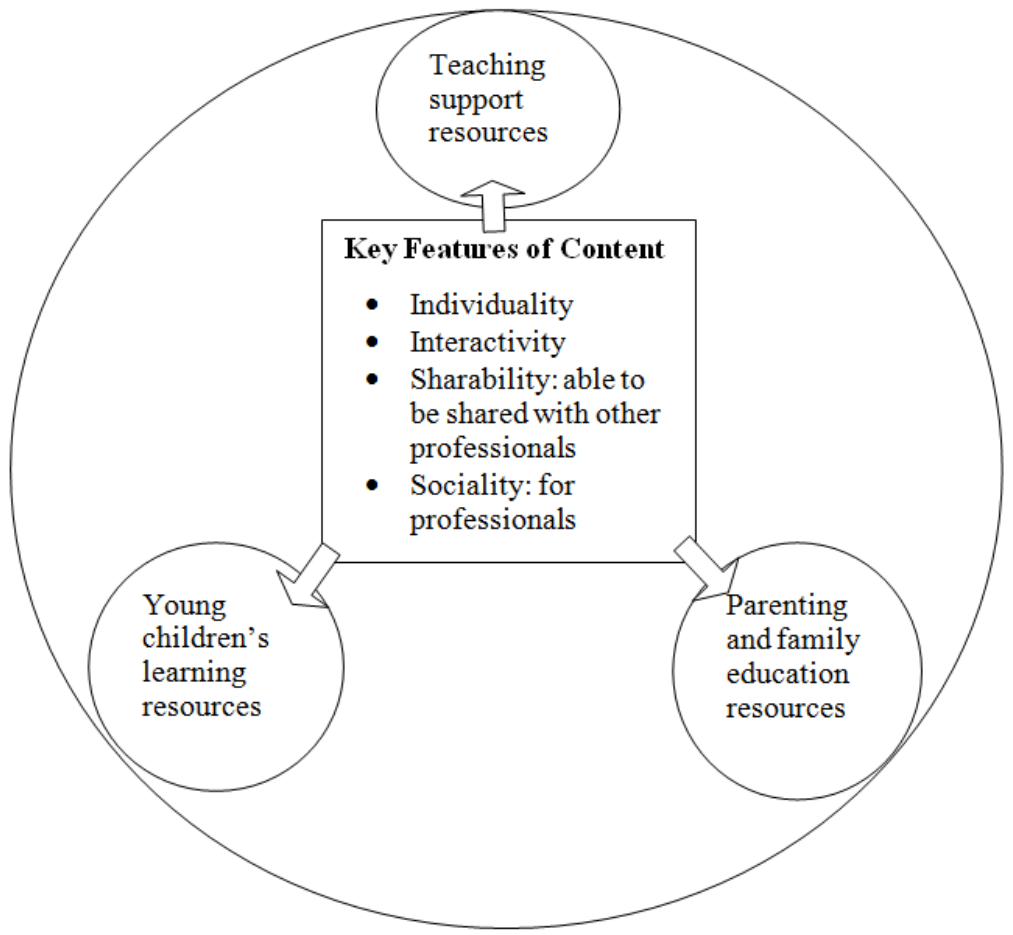

Figure 1. Key Elements in the ECE Corpus of E-Resources

Individuality of e-resources means that individual teachers can have the autonomy to access information from the corpus and produce their own individualized personal use of 
these resources for their particular classrooms. For instance, it may be feasible for teachers to set up their own tag system within the corpus rather than following a fixed system, or teachers can use Really Simple Syndication (RSS) to identify any information attracted to them and automatically transfer required information to their personal folders without being online or signing in to the corpus. This technology helps users classify information based on their personal needs and interests; this saves time and provides individualized services to users.

Interactivity refers to the use of blogs or other similar methods of online communication. This offers both the designers/producers and the users of the corpus the facility to publicize, edit and update information as well as to make comments and give feedback. The effect of online interaction is extremely valuable for ECE professionals, because it is fast, immediate and the time and place for communicating ideas are much less constrained. It is found that the use of a blog facilitates the knowledge management, teaching and research of ECE teachers and $87.5 \%$ of the participating teachers had a positive opinion towards the use of blogs for the professional development (Xiang \& Jiang, 2006).

The construction of the corpus of ECE e-resources may consider the model of Wikipedia to involve teacher users. This model gives opportunities to all users to participate and express their opinions. The sharability element becomes an important feature to glue users together. Many ECE teachers have their specialized and unique understanding, so they can share comments and possible solutions to many problems faced by teachers. To provide a platform to offer their ideas and share with users is an effective and time saving way to accumulate useful knowledge and obtain practical skills. This fits with both collective and individual values of using a corpus. However, one has to be aware that the freedom of editing and publicizing online could also affect the quality of e-resources and the accuracy of information. That information sometimes lacks authority and is unreliable is a common criticism of Wikipedia. Thus this ECE website may use the model but try to avoid the weaknesses of Wikipedia.

The sociality of using web contact is a value-added point for the development of this professionally focused corpus of e-resources. Teachers may use this web route to "meet" more professional friends and colleagues. They may help each other by exchanging and sharing new information, following up others' development and publishing their own new results or methods. This will assist the build-up inter-professional interaction and interaction between professionals and parents. In return, the e-resources will be expanded and enhanced.

The e-resource system for teaching support should contain classified information based on themes specified in the ECE curriculum. For example, all teaching materials and ECE textbooks have the theme of "animals"; this theme can be built up as a focus of information and teaching resources. Then teachers can search the relevant teaching materials within this theme to apply to their classroom practice, no matter what textbooks they are using. Teachers in a Shanghai kindergarten provided theme-based learning for a class of six year-olds by using e-resources and producing new teaching materials. They found that children showed their excitement, curiosity and eagerness in activities. Teachers used the e-resources to set up an e-environment with games and questions for the theme. These children were highly involved in the discussion, they interacted with online activities and were active in working with tasks and expressing their creative and individual opinions in group work. Their concentration span was much better than in traditional classes. The classroom activities aided by e-resources have stimulated both cognitive and affective factors for learning. In a kindergarten in Zhejiang province, 
teachers value the use of the same e-resources for different curriculum themes and different e-resources for the same theme, depending on the application of different phases of teaching activities.

It is also necessary to introduce the concept of knowledge domains for search functions. Taking animal as an example of theme again, when teachers need to search information and teaching resources of animals, different activities or domains may be designed to be linked with the theme so that teachers can search into, for instance, domains of language or music, in relation to animals. This will support teachers with richness and variety. Considering Chinese classroom teaching cultures for kindergartens, this e-resource should offer special support for activities for whole-class, small group, and individual teaching and learning. The classification of a large quantity of information into different domains helps improve the effectiveness of comprehension.

Ideally, the design of this corpus of e-resources would consider the management and functions to provide easy and speedy access to search, store and edit e-resources. Teachers are able to conduct searches, design activities and organize class activities, and evaluate their teaching as a related process of teaching and learning. In this way, this corpus will enhance its function for teaching and offer teachers opportunities to improve their teaching activities rather than constitute mainly a support tool.

The e-resource system for young children's learning should be differentiated from the current general e-games or study software for young children. This should follow the requirement of the ECE curriculum, to function as an extension of children's classroom learning and go beyond their classroom learning experience. This system should also include theme-focused design and provide venues of learning through play. Not only does it support young children with their classroom learning, but it also should aid them to learn independently. Teachers in a kindergarten in Zhejiang province have noticed that children participate more actively when the e-materials require them to use multiple senses (e.g. through music and colored pictures at the same time), and it appears that children remember such multi-sensory learning more effectively.

There have been controversies over the use of computers and the Internet for young children. However the reality is that children are using computers for learning. It is more important for us to study what and how they should learn through computers and the Internet. We may need to pay attention to appropriate methods and to the suitability of elearning based on the existing knowledge and experience we know about young children's learning methods and preferences. We may need to find out optimal amounts of time for young children to use computers for learning and at the same time, we need to cultivate a healthy environment for young children to use computers and the Internet following the ethics of e-learning.

The e-resource system for parent and family education is also an extension of kindergarten education; it provides a venue for parents to know how their child is learning and educated in a kindergarten. Since communication with parents is now increasingly emphasized in the ECE curriculum development, involving the participation of parents is a crucial part of kindergarten work. Through this e-resource system, parents can follow the progress of their child's learning and give home guidance to their child by understanding the ECE curriculum. 


\section{Conclusions}

In the current reform process of the Chinese ECE curriculum, professionals have begun to recognize the extensive and significant influences of IT and Internet resources to enhance teaching and learning ( $\mathrm{Li} \&$ Duan, 2005). The ever-growing development of Internet technology provides a workable model for constructing a corpus of e-resources for the ECE curriculum with features which can be made specifically suitable for and attractive to young learners. The corpus will also enhance the knowledge structure, professional skills and teaching competences of ECE teachers who are able to move towards more interactive and collaborative ways of teaching with the help of IT and eresources ( $\mathrm{Li}, 2008)$. Access to the corpus of e-resources will make ECE more economical and efficient; it can be constantly updated; it is sharable among professionals for different types of teaching and learning activities, such as for the whole class, small groups and individuals. It offers links between teachers, children, parents, communities and society together for mutually beneficial interaction and communication.

Kindergarten teaching in China will thus clearly benefit from the construction of a corpus of e-resources: professionals, learners and families will receive mutual benefits. However, at the same time, teachers face continuing challenges because they have to constantly update their technological skills and provisions to support the corpus and ultimately to serve users - teachers, children and parents. Both educational and IT professionals need to explore all possibilities and constantly analyze and meet the needs of users to develop new products using continuously updated technology to attract users and make this Chinese ECE corpus of e-resources sustainable.

The establishment and development of the Chinese ECE corpus of e-resources should seriously consider cultural teaching and learning features of the Chinese ECE. We learn from Western technology and useful theories and practices of ECE, but at the same time we should use our strengths in kindergarten practices, vast resources from Chinese ECE professionals and their teaching experiences to develop both technologically and professionally advanced corpus systems of e-resources which fit with Chinese cultures of ECE teaching and learning.

\section{References}

1 Chen, S., \& Zhou, J. (2009). You er yuan ke cheng shu zi hua zi yuan ku de shi yong yan jiu [Studies of the use of digital resources in kindergarten curriculum]. Early Childhood Education (Educational Science Edition), 447 \& 448, 10-13.

$2 \mathrm{Fu}$, N. (2008). Xin ke cheng shi shi zhong de jiao yu zi yuan xin xi hua [Digitalization of education resources in new curriculum practices]. China Information and Technology Education, 8, 113-114.

3 Guo, L. P., \& Qian, Q. (2006). Shanghai shi you er yuan ying yong xin xi ji shu de diao cha yu fen xi [The investigation and analysis of applying information technology in Shanghai kindergartens]. Shanghai Research on Education, 6, 37-40.

4 Guo, L. P., \& Wang, J. (2005). Ru he kan dai xin xi ji shu zai you er jiao yu zhong de ying yong [How to deal with the application of IT in early childhood education]. People's Education, 11, 20-21.

5 Jin, L., \& Cortazzi, M. (2006). Changing practices in Chinese cultures of learning, Language, Culture and Curriculum, 19(1), 5-20. 
6 Jin, L., \& Cortazzi, M. (2009). Culture et valeurs dans les classes chinoises [Classroom cultures and values: Students' conceptions of teachers and learning in China]. Revue Internationale d'Education de Sèvres, 50, 49-62.

7 Li, D. R., \& Duan, Z. B. (2005). Lun ke cheng zi yuan kai fa yu jiao shi zhuan ye cheng zhang [Discussion on the development of curriculum resources and teacher professional development]. Theory and Practice of Education, 6, 42-45.

8 Li. H. B. (2008). Xin xi wang luo zi yuan dui you er fa zhan ji xian xing ke cheng de ying xiang [Influences of Internet Resources to the Development of Young Children and Current Curriculum]. China Information and Technology Education, 4, 104-105.

9 The Ministry of Education of the People's Republic of China. (2002). You er yuan jiao yu zhi dao gang yao [The kindergarten education guidance]. Beijing: The Ministry of Education of the People's Republic of China

10 Xiang, X. Y., \& Jiang, Y. (2006). You er jiao shi zhuan ye fa zhan xin lu jing [A new path of professional development of ECE teachers: Blog and teacher development]. Preschool Education Study Journal, 7-8, 93-94.

11 Yan, H. M., \& Fan, W. (2006). Chu yi ke cheng zi yuan ji qi you xiao kai fa [Discussing curriculum resources and their development]. Theory and Practice of Education, 2, 38-40.

12 Zhou, J., \& Chen, S (2009). You er yuan ke cheng shu zi hua zi yuan ku de gong neng he jia zhi [Functions and values of digital resources in kindergarten curriculum]. Early Childhood Education (Educational Science Edition), 429 \& 430. 15-19. 